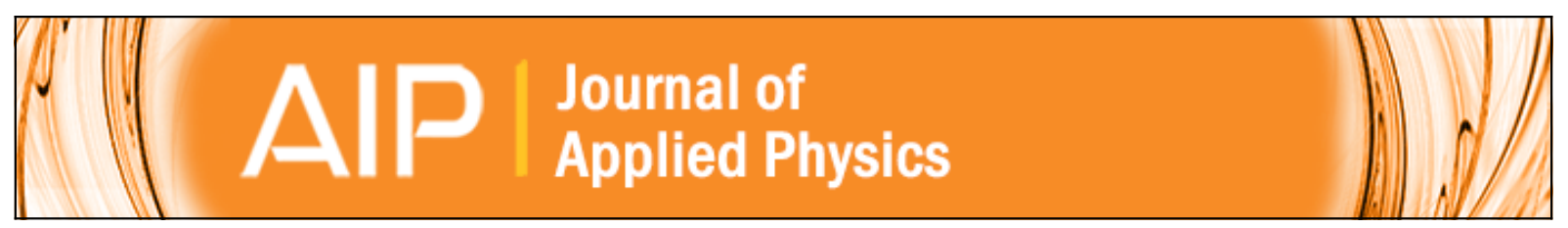

\title{
Magnetic structuring of linear copper electrodeposits
}

Peter Dunne, Rémy Soucaille, Karl Ackland, and J. M. D. Coey

Citation: Journal of Applied Physics 111, $07 B 915$ (2012); doi: 10.1063/1.3678295

View online: http://dx.doi.org/10.1063/1.3678295

View Table of Contents: http://scitation.aip.org/content/aip/journal/jap/111/7?ver=pdfcov

Published by the AIP Publishing

\section{Articles you may be interested in}

Electrodeposition of $\mathrm{Au} / \mathrm{Ag}$ bimetallic dendrites assisted by Faradaic AC-electroosmosis flow

AIP Advances 4, 031329 (2014); 10.1063/1.4868518

Nanostructure and oxide phase distribution in Co36-40Fe64-60 electrodeposited films for magnetic field sensors J. Appl. Phys. 111, 07A330 (2012); 10.1063/1.3677931

Tuning magnetic hysteresis of electrodeposited Fe 304

J. Appl. Phys. 98, 113902 (2005); 10.1063/1.2135892

Photoexcited electrodeposition of Cu structures on $\mathrm{p}$ - Si (001)

J. Vac. Sci. Technol. A 22, 1842 (2004); 10.1116/1.1690257

Structural and magnetic fourfold symmetry of $\mathrm{Co} / \mathrm{Cu}$ multilayers electrodeposited on $\mathrm{Si}(001)$ substrates J. Appl. Phys. 84, 1504 (1998); 10.1063/1.368254

\section{High-Voltage Amplifiers}

-Voltage Range from $\pm 50 \mathrm{~V}$ to $\pm 60 \mathrm{kV}$

- Current to $25 \mathrm{~A}$

\section{Electrostatic Voltmeters}

- Contacting \& Non-contacting

- Sensitive to $1 \mathrm{mV}$

- Measure to $20 \mathrm{kV}$
ENABLING RESEARCH AND

INNOVATION IN DIELECTRICS,

ELECTROSTATICS, MATERIALS, PLASMAS AND PIEZOS

Rek www.trekinc.com 


\title{
Magnetic structuring of linear copper electrodeposits
}

\author{
Peter Dunne, Rémy Soucaille, Karl Ackland, and J. M. D. Coey ${ }^{a)}$ \\ School of Physics and CRANN, Trinity College, Dublin 2, Ireland
}

(Presented 2 November 2011; received 12 September 2011; accepted 25 November 2011; published online 9 March 2012)

\begin{abstract}
Electrodeposition of copper is investigated in localized magnetic fields produced by linear arrays of permanent magnets. The thickness and texture of the deposits depend on the magnitude and direction of the field. The deposition rate is explained in terms of magnetic pressure on the diffusion layer. Addition of non-electroactive $\mathrm{GdCl}_{3}$ to the electrolyte inverts the structuring of the electrodeposits, producing thick dendritic growth in regions where the field is smallest. () 2012 American Institute of Physics. [doi:10.1063/1.3678295]
\end{abstract}

Electrodeposition is widely used method to prepare thick films of ferromagnetic metals and alloys such as permalloy for use as cores or shields in thin film heads ${ }^{1}$ or cores in on-chip inductors. ${ }^{2}$ The application of a magnetic field during deposition of these ferromagnetic metals or alloys may introduce crystallographic or atomic-scale texture, which can influence the anisotropy and magnetic reversal in the electrodeposited material. ${ }^{3-5}$

More recently, there have been reports of structuring of electrodeposits from paramagnetic solutions such as $\mathrm{Cu}^{2+}$ using nonuniform magnetic fields produced by permanent magnets, ${ }^{6,7}$ or soft iron, which is magnetized in an external field. ${ }^{8,9}$ These studies establish that it is the magnetic susceptibility of the electroactive species, not the susceptibility of the electrolyte itself, which determines the pattern. ${ }^{7,10}$ The deposits are controlled by the magnitude of the field at the cathode and its gradient across the surface. Permanent magnets are particularly suitable for generating magnetic fields, which vary over a short length scale. Inverse patterns where the electrodeposit builds up in regions where the magnitude of the field at the surface is smallest, have been observed when a strongly paramagnetic but non-electroactive species, such as a trivalent rare earth ion, is added to the electrolyte. ${ }^{7,11}$

We have recently given a detailed account of how the normal and inverse electrodeposits are influenced by a variety of different arrays of small, cylindrical permanent magnets. $^{12}$ Here we report on the normal and inverse electrodeposition of copper when permanent magnet line arrays are used to generate the nonuniform stray fields at the cathode surface.

Two different baths were used, one for direct deposition, the other for inverse deposition. Their compositions were:

$$
\begin{aligned}
\text { Bath 'A' } \quad & 0.1 \mathrm{M} \mathrm{CuSO}_{4}+1 \mathrm{M} \mathrm{Na}_{2} \mathrm{SO}_{4} \\
& +0.1 \mathrm{M} \mathrm{CH}_{3} \mathrm{COOH}+0.1 \mathrm{M} \mathrm{NaCH}_{3} \mathrm{COO},
\end{aligned}
$$

$$
\text { Bath 'B' } \quad 0.1 \mathrm{M} \mathrm{CuSO}_{4}+1 \mathrm{M} \mathrm{GdCl}_{3}+0.4 \mathrm{MH}_{3} \mathrm{BO}_{3} \text {. }
$$

\footnotetext{
${ }^{\text {a) }}$ Author to whom correspondence should be addressed. Electronic mail: jcoey@tcd.ie.
}

The composition of bath ' $\mathrm{A}$ ' is chosen to avoid the formation of hydrogen bubbles at the overpotential used for deposition, $-0.85 \mathrm{~V}$ relative to $\mathrm{Ag} / \mathrm{AgCl}$. The overall susceptibility of the bath, $\chi_{\mathrm{A}}=-7 \times 10^{-6}$, is due mainly to the diamagnetism of water, $\chi_{\text {water }}=-9 \times 10^{-6}$. The susceptibility of the $\mathrm{Cu}^{2+}$ solution $(\mathrm{S}=1 / 2, \mathrm{~g}=2)$ is $1.6 \times 10^{-6}$. The susceptibility $\chi_{\mathrm{B}}$ of bath ' $\mathrm{B}^{\prime}$ is dominated by the paramagnetism of the $1 \mathrm{M}$ $\mathrm{Gd}^{3+}$ solution $(\mathrm{S}=7 / 2, \mathrm{~g}=2) ; \chi=320 \times 10^{-6}$.

Our electrochemical cell has a volume of $60 \mathrm{~cm}^{3}$, and is not significantly depleted of copper in the course of the experiments. The working electrode is a silver foil $50 \mu \mathrm{m}$ thick, which is backed either by a single rectangular magnet $5 \times 5 \times \tau \mathrm{mm}^{3}$ magnetized parallel to the short direction, placed near the center of the foil, or by an array of lines of these magnets. The arrays were made up in templates with a series of regularly spaced slots. Magnet widths were $\tau=0.5$, 1.0 , or $1.2 \mathrm{~mm}$, and the center-to-center separation was 1.5 , 2.5 , or $3.0 \mathrm{~mm}$, respectively. The diameter of the working electrode exposed to the solution was $19 \mathrm{~mm}$, but the magnet arrays were larger to minimize edge effects. Alternate lines are magnetized in opposite directions, parallel to the electrode surface, in order to create the maximum stray field at the cathode surface. Figure $1,{ }^{7,12}$ shows the calculated field contours for the perpendicular and parallel components of the magnetic field there.

Deposits were photographed using a USB microscope camera, and many were examined in a Zeiss Auriga dualbeam scanning electron microscope (SEM) where cross sections were excised by $\mathrm{Ga}^{+}$ion-beam (FIB) milling.

Images of the direct copper deposits obtained at -0.85 $\mathrm{V}$ for $600 \mathrm{~s}$ from bath ' $\mathrm{A}$ ' are shown in Fig. 2. The regions between the magnets, where the field is perpendicular to the cathode appear bright and the regions below the magnets where the field is parallel appear dark. However, the contrast can be deceptive because it varies with the angle of illumination. The light scattering is related to the different texture of the copper deposited in the two regions, as seen in Fig. 2(b). The FIB cross sections in Fig. 2(c) show that the deposit is about twice as thick in the $180 \mathrm{mT}$ perpendicular field between the magnet lines $(350 \mathrm{~nm})$ as it is in the $300 \mathrm{mT}$ parallel field $(\sim 0 \mathrm{mT}$ perpendicular field) below them $(180 \mathrm{~nm})$ [Fig. 2(b)]. Similar deposits are found for greater copper 


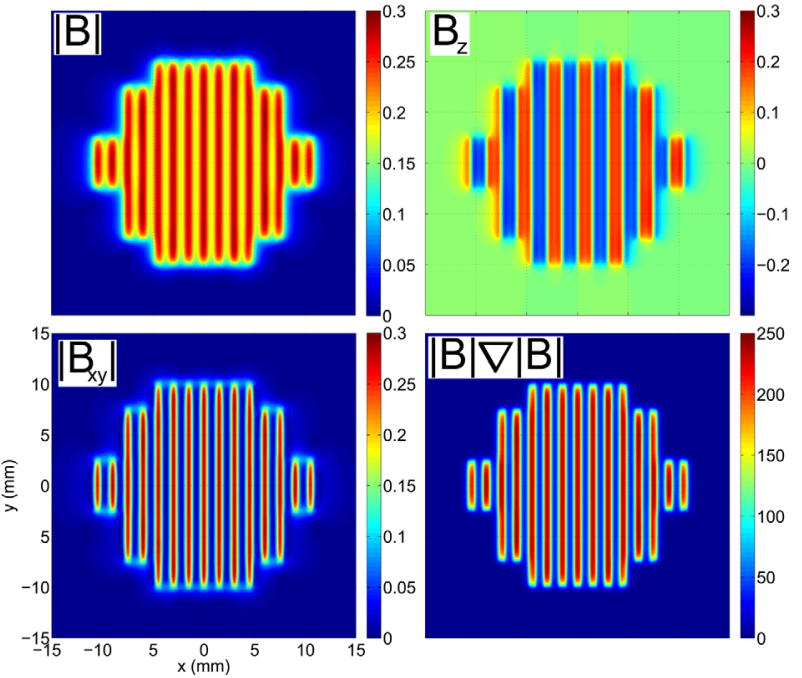

FIG. 1. (Color online) Calculated profiles of the total, perpendicular and inplane magnetic field at the cathode surface for the $0.5 \mathrm{~mm}$ linear array. The field gradient profile $1 / 2 \nabla \mathrm{B}^{2}$ is also shown.

concentrations, $0.56 \mathrm{M}$ (zero net susceptibility) or $1.0 \mathrm{M}$, confirming that it is the susceptibility of the electroactive copper and not that of the bulk electrolyte that counts. ${ }^{7,10}$

An estimate of the forces involved in the structuring is obtained by changing the orientation of the cathode relative to gravity. When the cathode is downward-facing, as it is in Fig. 3, no convection is driven directly by the density decrease $\Delta \rho \approx 15 \mathrm{~kg} \mathrm{~m}^{-3}$ when the electrolyte is depleted of copper, and the current decays continually with time. But when the cathode faces upwards or is vertical with horizontal magnetic stripes, the deposition current rapidly stabilizes at a high value $(\sim 18 \mathrm{~mA})$ but the structure is smeared out by convection, which indicates that the magnetic forces involved in the patterning of the electrodeposit are of order $150 \mathrm{~N} \mathrm{~m}^{-3}$. However, when the cathode is vertical with vertical magnetic stripes, the patterning is retained at an enhanced deposition rate.

Electrodeposits for the $\mathrm{Cu} / \mathrm{Gd}$ bath ' $\mathrm{B}$ ' are quite different. With a single magnet, the deposits are thick and dendritic far from the magnet where the field is zero, and they are thinner and shiny near the magnet. With a line array the deposit is very rough everywhere, but thicker in between the magnets and thinner beneath them.

Variations in the equilibrium concentration of copper associated with the fluctuations in magnitude of the magnetic field $B$ near the cathode are miniscule. The energy at room temperature of a single ion of spin $S$ at temperature $T$, $\mathrm{g}^{2} \mu_{\mathrm{B}}^{2} S(S+1) B^{2} / \mathrm{k} T$, is 5 or 6 orders of magnitude less than $\mathrm{k} T$, the energy which drives diffusion and determines the equilibrium concentration. The equilibrium is dynamic, not static.

The electrodeposition of copper at $-0.85 \mathrm{~V}$ is masstransport limited. The current density $j=2 \mathrm{~F} D \nabla c$, where $\mathrm{F}$ is Faraday's constant, $D$ is the diffusion constant of $\mathrm{Cu}^{2+}$ and $\nabla c=c_{0} / \delta$, where $c_{0}=100 \mathrm{~mol} \mathrm{~m}^{-3}$ is the bulk copper concentration and $\delta$ is the thickness of the diffusion layer that controls the mass transport. ${ }^{13}$ For the downward-facing cathode, the current transient is uninfluenced by natural convection, and the value of $D=0.5 \times 10^{-9} \mathrm{~m}^{2} \mathrm{~s}^{-1}$ is deduced for
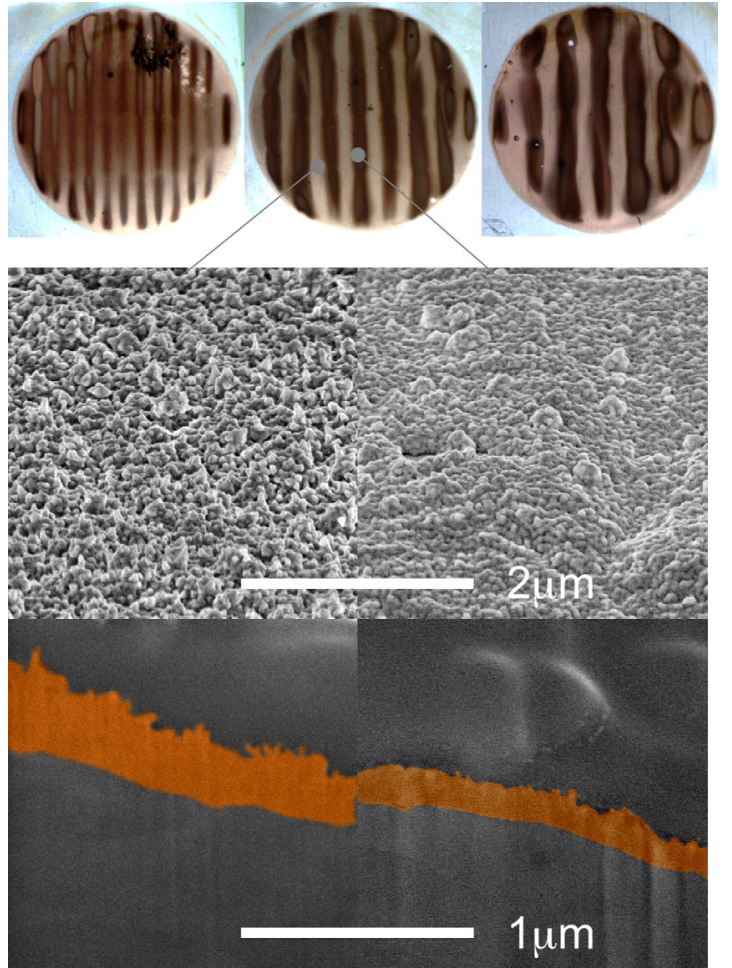

FIG. 2. (Color online) (top) Electrodeposits from bath A with different magnet line arrays. (middle) SEM images of the deposits in the darker regions between the magnets and in the lighter regions beneath them. Cross sections are shown in the bottom panel. A strip of Pt was deposited to help protect the electrodeposit when milling a trench with the focused ion beam.

bath ' $\mathrm{A}$ ' from the time-dependent current density in Fig. 3 using the Cottrell equation $j(t)=2 \mathrm{~F} c_{0}(D / \pi t)^{1 / 2} \cdot{ }^{13}$ The diffusion layer thickness deduced from the average current of 5.5 $\mathrm{mA}$ for the downward-facing cathode (area $2.8 \times 10^{-4} \mathrm{~m}^{2}$ ) is therefore $500 \mu \mathrm{m}$, whereas the limiting current of about 18 $\mathrm{mA}$ for the upward-facing cathode gives $\delta=153 \mu \mathrm{m}$. These values assume the diffusion layer thickness is uniform. The variation in deposit thickness indicates otherwise.

The Lorentz force density $\mathbf{F}_{\mathrm{L}}=\mathbf{j} \times \mathbf{B}$ is unimportant in our experiments; for a current density of $20 \mathrm{~A} \mathrm{~m}^{-2}$ it amounts to just $6 \mathrm{~N} \mathrm{~m}^{-3}$ in the horizontal field beneath the magnet lines. Further, the induced flow will be frustrated by the pattern of alternating magnetization. The force is larger when the current density is increased by convection to about $60 \mathrm{~A} \mathrm{~m}^{-2}$, but it remains less than the mean force density driving convection in the diffusion layer $[1 / 2] \Delta \rho \mathrm{g}=75 \mathrm{~N} \mathrm{~m}^{-3}$. The limiting current for the upwardfacing cathode is observed to increase by only $15 \%$ in a uniform horizontal field of $500 \mathrm{mT}$.

The effects we observe require a magnetic field gradient and an orthogonal concentration gradient. ${ }^{12}$ The gradient force on the paramagnetic ions may be written as $F_{\nabla \mathrm{B}}=\left(1 / 2 \mu_{0}\right) \chi_{\mathrm{mol}} c \nabla B^{2}$. When $\nabla B^{2}=250 \mathrm{~T}^{2} \mathrm{~m}^{-1}$, this force is $180 \mathrm{~N} \mathrm{~m}^{-3}$ for $0.1 \mathrm{M} \mathrm{Cu}^{2+}$ and $3 \times 10^{4} \mathrm{~N} \mathrm{~m}^{-3}$ for $1 \mathrm{M} \mathrm{Gd}^{3+}$. It pales by comparison with the effective force density driving diffusion, which is $\mathrm{R} T \nabla c \approx 5 \times 10^{8} \mathrm{~N} \mathrm{~m}^{-3}$, but $F_{\nabla \mathrm{B}}$ is able to control convection and advection. ${ }^{14}$ Note that $\left|B_{\mathrm{z}}\right|$ is zero below the central magnet line in the array of Fig. 1, but the other three contours are maximum there. This 

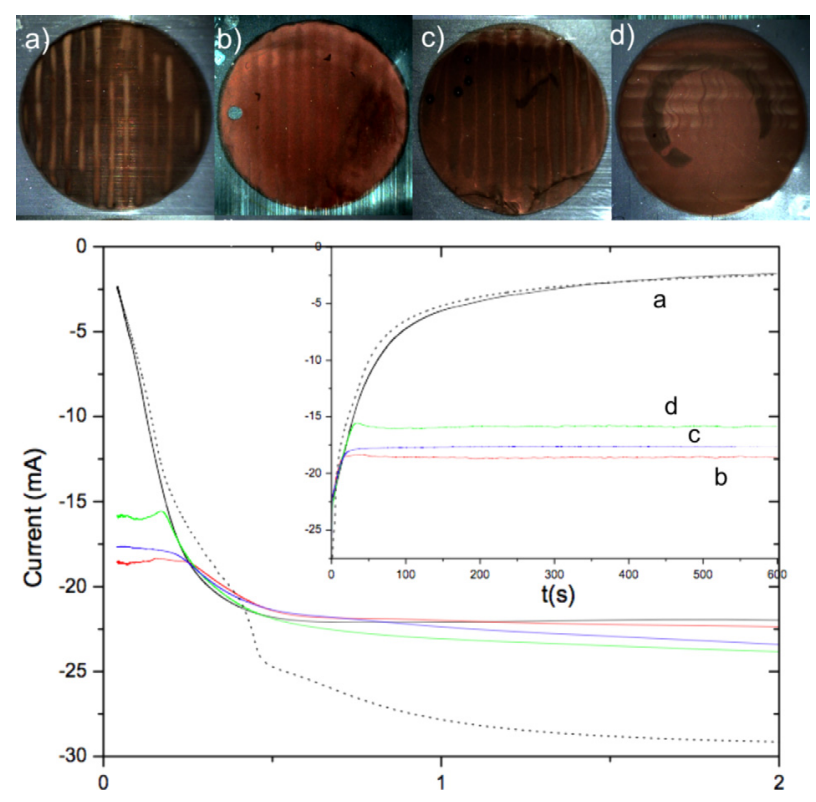

FIG. 3. (Color online) Electrodeposits obtained from bath A with the $\tau=0.5 \mathrm{~mm}$ linear magnet array when the cathode faces (a) downward, (b) upward or sideways with the lines (c) vertical or (d) horizontal. The graph shows the current as a function of and $\mathrm{t}^{-1 / 2}$ and time $\mathrm{t}$ for the different orientations, and without the magnet array (dashed lines).

observation suggests that it is $\left|B_{z}\right|$ that primarily influences the deposition.

So how exactly does the nonuniform magnetic field lead to structuring of the electrodeposits? The answer is by magnetic pressure on the diffusion layer, which can be calculated from the force on the induced magnetic charge density there. This was the dominant effect for the patterning produced by small cylindrical magnets, where the field is largely perpendicular to the cathode. ${ }^{12}$ The volume charge density is $\nabla \cdot \mathbf{M}=\mathrm{d}\left[\chi_{\mathrm{mol}} c(\mathrm{z}) \mathrm{B}_{\mathrm{z}} / \mu_{0}\right] / \mathrm{dz}$. As $c(\mathrm{z})=c_{0} \mathrm{z} / \delta$ for $\mathrm{z} \leq \delta$ and $c_{0}$ for $\mathrm{z} \geq \delta$, the magnetic force density in the diffusion layer is $\chi_{\mathrm{mol}} c_{0} \mathrm{~B}_{\mathrm{z}}^{2} / \mu_{0} \delta$ and the corresponding pressure is $\chi_{\mathrm{mol}} c_{0} \mathrm{~B}_{\mathrm{z}}^{2} / 2 \mu_{0}$, where $\chi_{\mathrm{mol}} c_{0} \mathrm{~B}_{\mathrm{z}} / 2 \mu_{0}$ is the field-averaged magnetization assuming the field is uniform there. ${ }^{15}$ There is no force when $c$ is uniform because $\mathbf{M}$ is then proportional to $\mathbf{B}$, and $\nabla \cdot \mathbf{M}=\nabla \cdot \mathbf{B}=0$. The pressure is compensated by fluctuations $\Delta \delta$ in the thickness of the diffusion layer as $B_{\mathrm{z}}$ varies across the cathode, giving a pressure difference $[1 / 2] \Delta \rho \mathrm{g} \Delta \delta$. For example, for $0.1 \mathrm{M} \mathrm{CuSO}{ }_{4}$ when $B_{\mathrm{Z}}=180 \mathrm{mT}$, the pressure is $21 \mathrm{~N} \mathrm{~m}^{-3}$ and $\Delta \delta=276 \mu \mathrm{m}$. If the average value of $\delta$ deduced from the average current density is $500 \mu \mathrm{m}$, the difference of $276 \mu \mathrm{m}$ corresponds to diffusion layer thicknesses of $398 \mu \mathrm{m}$ between the magnets and $674 \mu \mathrm{m}$ below them, assuming equal areas of the two regions, giving an estimated thickness ratio of 1.7:1, which agrees with observation. The estimate is a rough one, because little patterning occurs in the early stages when the diffusion layer is being set up, or in the late stages when $\delta$ exceeds the spacing of the magnets, but it shows that magnetic pressure can account for the magnitude of the effect. The curl $\nabla \times \mathbf{F}_{\nabla \mathrm{B}}=\left(1 / 2 \mu_{0}\right) \chi_{\mathrm{mol}}\left(\nabla \mathrm{c} \times \nabla \mathrm{B}^{2}\right)$ shows that the force is effective at inducing flow to structure the diffusion layer when a lateral field gradient acts on a vertical concentration gradient, and vice versa.

The effect of adding strongly paramagnetic gadolinium to the electrolyte is to impart a huge effective diamagnetic susceptibility to the electroactive copper, ${ }^{16}$ and copperdepleted aqueous solution. This greatly enhances the forces acting on the copper and changes their sign. With a single magnet, the thick, dendritic deposit builds up far from the magnet, where the field is zero, but with a line array, the deposit builds up between the magnets, where the magnitude of the field is smaller.

In conclusion, we have shown how to use magnetic fields to pattern metal electrodeposits using electrolytes containing electroactive or non-electroactive paramagnetic ions. The principle involved for direct deposits is modification by magnetic pressure of the diffusion layer thickness, and this that limits the scale on which it is possible to observe the effects. At best, structures on the scale of tens of microns can be anticipated. The quality of the deposits may be improved using recognized additives in the baths. Patterning of ferromagnetic metals and alloys is possible with this method, but the analysis is complicated by the deformation of the local flux density by the deposited ferromagnetic material.

This research was supported by Science Foundation Ireland as part of the NISE project. The work was conducted in the framework of the Irish government PRTLI-4 program. K.A. was supported by IRCSET.

${ }^{1}$ E. L. Cooper et al, IBM J. Res. Dev. 49, 103 (2005).

${ }^{2}$ S. C. O'Mathuna, T. O'Donnell, N.-N. Wang, and K. Rinne, IEEE Trans. Power Electron. 20, 585 (2005).

${ }^{3}$ E. Kubo, N. Ooi, H. Aoki, D. Watanabe, J.-H. Jeong, C. Kimura, and T. Sugino, Jpn. J. Appl. Phys. 49, 04DB17 (2010).

${ }^{4}$ I. Tabakovic, S. Reimer, V. Vasko, V. Sapozhnikov, and M. Kief, J. Electrochem. Soc. 150, 635 (2003).

${ }^{5}$ K. Msellak, J.-P. Chopart, O. Jbara, O. Aaboubi, and J. Ambelard, J. Magn. Magn. Mater. 281, 295 (2004).

${ }^{6}$ G. Mutschke, K. Tschulik, T. Weier, M. Uhlemann, A. Bund, and J. Fröhlich, Electrochim. Acta 55, 9060 (2010).

${ }^{7}$ P. Dunne, L. Mazza, and J. M. D. Coey, Phys. Rev. Lett. 107, 024501 (2011).

${ }^{8}$ K. Tschulik, J. A. Koza, M. Uhlemann, A. Gerbert, and L. Schultz, Electrochem. Commun. 11, 2241 (2009).

${ }^{9}$ K. Tschulik, R. A. Sueptitz, M. Uhlemann, L. Schultz, and A. Gerbert, Electrochim. Acta 56, 5174 (2011).

${ }^{10}$ K. Tschulik, R. Sueptitz, J. Koza, M. Uhlemann, G. Mutschke, T. Weier, A. Gerbert, and L. Schultz, Electrochim. Acta 56, 297 (2010).

${ }^{11}$ K. Tschulik et al, Electrochem. Commun. 13, 946 (2011).

${ }^{12}$ P. A. Dunne, PhD thesis, Dublin 2011.

${ }^{13}$ C. M. A. Brett and A. M. Oliveira Brett, Electrochemistry, Principles, Methods and Applications (Oxford University Press, Oxford, 1992).

${ }^{14}$ J. M. D. Coey, R. Aogaki, F. Byrne, and P. Stamenov, Proc. Natl. Acad. Sci. U.S.A. 106, 8811 (2009).

${ }^{15}$ R. E. Rosensweig, Ferrohydrodynamics (Dover, New York, 1997).

${ }^{16}$ P. A. Dunne, J. Hilton, and J. M. D. Coey, J. Magn. Magn. Mater. 316/2, 273 (2007). 